\title{
Radio frequency noise effects on the CERN Large Hadron Collider beam diffusion
}

\author{
T. Mastoridis, * P. Baudrenghien, A. Butterworth, and J. Molendijk \\ BE-RF, CERN, Geneva, Switzerland \\ C. Rivetta ${ }^{\dagger}$ and J. D. Fox \\ SLAC National Accelerator Laboratory, Stanford, California 94309, USA
}

(Received 5 April 2011; published 26 September 2011)

\begin{abstract}
Radio frequency (rf) accelerating system noise can have a detrimental impact on the Large Hadron Collider (LHC) performance through longitudinal motion and longitudinal emittance growth. A theoretical formalism has been developed to relate the beam and rf station dynamics with the bunch length growth [T. Mastorides et al., Phys. Rev. ST Accel. Beams 13, 102801 (2010)]. Measurements were conducted at LHC to determine the performance limiting rf components and validate the formalism through studies of the beam diffusion dependence on rf noise. As a result, a noise threshold was established for acceptable performance which provides the foundation for beam diffusion estimates for higher energies and intensities. Measurements were also conducted to determine the low level rf noise spectrum and its major contributions, as well as to validate models and simulations of this system.
\end{abstract}

DOI: 10.1103/PhysRevSTAB.14.092802

PACS numbers: $29.20 . \mathrm{db}, 29.27 . \mathrm{Bd}$

\section{INTRODUCTION}

The synchrotron radiation damping in a hadron accelerator is usually very low. Particles in the Large Hadron Collider (LHC) lose $7 \mathrm{keV}$ per turn for the nominal energy of $7 \mathrm{TeV}$. During a long store, the relationship between the energy lost to synchrotron radiation and the noise injected to the beam by the rf accelerating voltage determines the growth of the longitudinal energy spread and longitudinal emittance. As a result, beam diffusion is extremely sensitive to $\mathrm{rf}$ perturbations.

The theoretical formalism presented in [1] suggests that the noise experienced by the beam depends on the accelerating voltage phase noise power spectrum, aliased to a band between DC and the first revolution harmonic, due to the periodic sampling of the accelerating voltage $V_{c}$ by the beam. Additionally, the dependence of the rf accelerating voltage noise spectrum on the low level rf (LLRF) configurations has been predicted using time-domain simulations and models described in [2]. In this work, measurements at the LHC supporting the above theoretical formalism and simulation predictions are presented. A noise threshold for acceptable LHC performance is then estimated.

Section II briefly describes the rf system with an emphasis on beam diffusion. The theoretical formalism of the beam diffusion dependence on rf noise is briefly presented in Sec. III. Section IV presents the noise-generating rf

\footnotetext{
*themis.m@cern.ch; formerly known as T. Mastorides.

†rivetta@slac.stanford.edu
}

Published by the American Physical Society under the terms of the Creative Commons Attribution 3.0 License. Further distribution of this work must maintain attribution to the author(s) and the published article's title, journal citation, and DOI. components, and identifies the element that dominates the longitudinal beam emittance blowup effects. Quantitative experiments of the beam diffusion dependence on rf noise are shown in Sec. V. Based on these measurements, a noise threshold for acceptable lifetime in the LHC is presented in Sec. VI. Section VII studies the LLRF noise dependence on the controller gain settings and compares the LLRF noise spectrum estimated by the timedomain simulations with data from LHC. Finally, Sec. VIII includes an elementwise study of the rf feedback noise contributions. This paper follows the work previously presented by the authors in $[1,3]$.

\section{SYSTEM DESCRIPTION}

The LHC rf system consists of eight rf stations per beam. The rf system accelerates the beam during the ramp, compensates the small energy losses during coasting, and also provides longitudinal focusing. A simplified block diagram of the LHC rf system is shown in Fig. 1.

Each rf station includes an accelerating superconducting cavity, a $330 \mathrm{~kW}$ klystron, and the LLRF system consisting of the klystron polar loop and the impedance control feedback system. The superconducting cavity has an $R / Q$ of $45 \Omega$, a resonance frequency of $400.8 \mathrm{MHz}$, and a mechanical tuner with a $100 \mathrm{kHz}$ range. For nominal intensity beams, the cavity voltage and loaded quality factor $Q_{L}$ are set to $1 \mathrm{MV}$ and 20000 , respectively, during injection (flat bottom) and to $2 \mathrm{MV}$ and 60000 during collision (flattop). The LLRF feedback system acts to reduce the rf station fundamental impedance as sampled by the beam and increase longitudinal stability. It incorporates digital and analog paths, as well as the one-turn feedback (comb), which acts to reduce the impedance at the revolution harmonics. The klystron polar loop as implemented at 


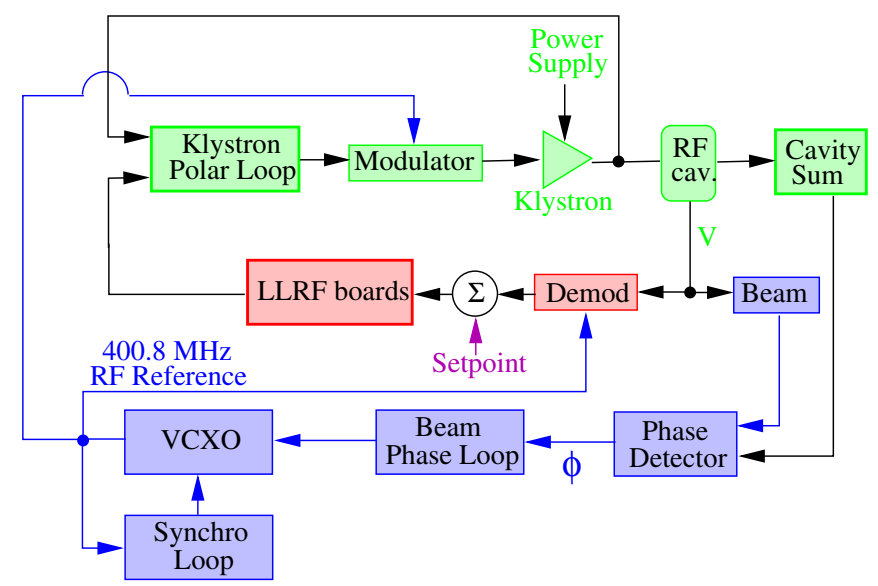

FIG. 1. Simplified block diagram of the rf system with beam phase loop.

the LHC acts to stabilize the klystron gain and phase response against variations due to power supply fluctuations and operation point changes.

The LLRF feedback system processing takes place in baseband. A $400.8 \mathrm{MHz}$ rf reference signal is created by the voltage-controlled crystal oscillator (VCXO) to modulate/demodulate the baseband signals to/from rf frequencies. The VCXO is controlled by the synchroloop and the beam phase loop (BPL). The BPL is a narrow bandwidth loop which acts on the VCXO to damp out barycentric longitudinal motion around the synchronous phase, motion driven by noise in the rf system or by other mechanisms. The BPL uses the individual bunch phases averaged over one turn. It sends a correction to the VCXO once per turn, and therefore has no gain above the revolution frequency $f_{\text {rev }}$ of $11.245 \mathrm{kHz}$.

The input to the BPL is the beam phase error signal $\phi$, a measure of the average deviation of the phase difference between the beam and the cavity sum over a turn. The cavity sum represents the total accelerating voltage per

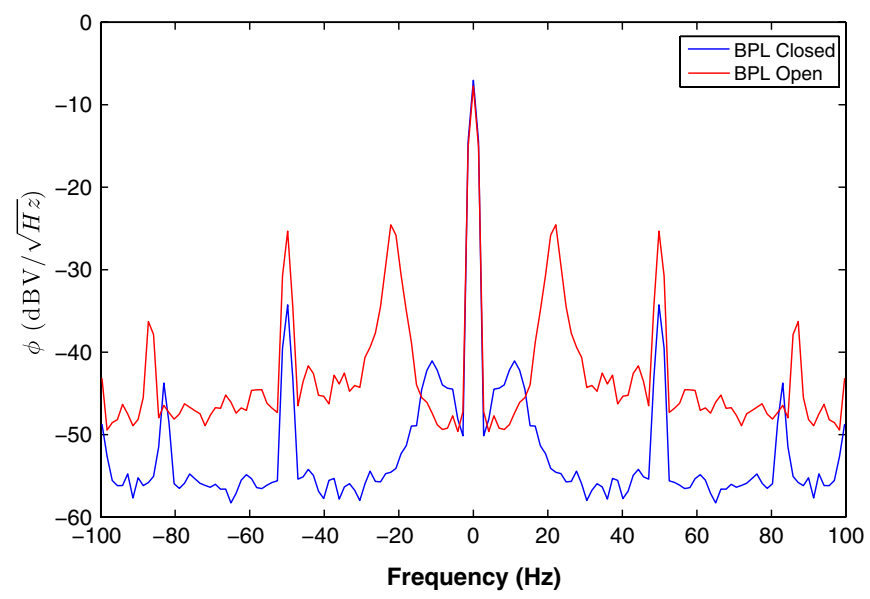

FIG. 2. Beam 1 phase error with beam phase loop open/closed. beam. The BPL controls the phase of the VCXO so that $\phi$ is reduced around the synchrotron frequency $f_{s}$. The BPL includes an adjustable gain. The substantial reduction in longitudinal mode zero with the BPL can be seen in Fig. 2, which shows the spectrum of $\phi$ with the BPL open or closed. With the BPL open there is a substantial phase difference between the beam and the cavity sum at the synchrotron frequency of about $23 \mathrm{~Hz}$, due to the synchrotron oscillation. The lines at $50 \mathrm{~Hz}$ and harmonics seen in the figure are from the AC main power supplies and are artifacts of the instrumentation system.

\section{BEAM DIFFUSION DEPENDENCE ON RF NOISE}

Under nominal operating conditions $\left(1.15 \times 10^{11}\right.$ particles per bunch), bunches of initial length ${ }^{1}$ of about $375 \mathrm{ps}$ would be injected into the LHC and reduced to about $250 \mathrm{ps}$ during the energy ramp. During the long store, the bunch diffuses longitudinally due to intrabeam scattering (IBS) and rf noise. The Fokker-Planck formalism is used often to describe the latter effect [4]. This formalism cannot be applied to colored noise sources [5] and does not include the aliasing effect due to the beam periodicity.

The particle beam samples the cavity phase noise $\phi_{\text {cav }}(t)$ every revolution harmonic, so that

$$
\tilde{\phi}(t)=\sum_{k=-\infty}^{\infty} \delta\left(t-k T_{o}\right) \phi_{\mathrm{cav}}(t)
$$

where $T_{o}$ is the revolution period and $\tilde{\phi}(t)$ is the phase noise experienced by the beam. Since the beam is a very high $Q$ resonator at the synchrotron frequency $f_{s}$, the beam sampled power $P_{n}$ is dominated by the noise power spectral density around $k f_{\text {rev }} \pm m f_{s}$ where $f_{\text {rev }}$ is the revolution frequency, $k$ an integer, and $m$ is the azimuthal mode number ( $m=1$ for dipole modes, $m=2$ for quadrupole modes, etc.). In this work we focus on $m=1$, since this mode dominates the diffusion of the bunch core, with the LHC bunch length (250-375 ps) small compared to the bucket width of 675 ps.

Following [6,7], the bunch length growth rate can be estimated by

$$
\frac{d \sigma_{\tau}^{2}}{d t}=\frac{d \sigma_{\phi}^{2}}{\omega_{\mathrm{rf}}^{2} d t}=\frac{\omega_{s}^{2}}{2 \pi \omega_{\mathrm{rf}}^{2}} \sum_{k=-\infty}^{\infty} S_{\phi}\left(k f_{\mathrm{rev}} \pm f_{s}\right)=2 \sigma_{\tau} \frac{d \sigma_{\tau}}{d t},
$$

where $\omega_{\mathrm{rf}}$ is the $\mathrm{rf}$ angular frequency, $\omega_{s}=2 \pi f_{s}$ is the angular synchrotron frequency, and $S_{\phi}(f)$ is the accelerating cavity phase noise spectral density (in $\operatorname{rad}^{2} / \mathrm{Hz}$ ).

\footnotetext{
${ }^{1}$ In this work, the bunch length is defined as the "root-mean square" value of the longitudinal particle distribution in one $\mathrm{rf}$ bucket $\left(\sigma_{\tau}\right)$.
} 


\section{RF NOISE DOMINATING COMPONENTS}

Before studying the effect of the accelerating voltage phase noise on the longitudinal beam emittance, it is important to determine the rf components that impact the accelerating voltage noise spectrum. The high gain rf feedback and the klystron polar loop compensate for all sources of cavity (tune fluctuation due to helium pressure) and klystron noise (high voltage ripple), so that the major remaining noise contributions are from the LLRF signal processing electronics and the rf reference oscillator signal. It should be noted that the rf reference noise is correlated over all cavities, whereas the LLRF noise is uncorrelated with the exception of noise related to the $50 \mathrm{~Hz}$ power supply ripple.

Initial measurements of the power spectral density of the accelerating voltage phase noise during operations with $3.5 \mathrm{TeV}$ beam and the BPL open showed that the phase noise is dominated by the rf reference up to $\approx 300 \mathrm{~Hz}$, and the LLRF controller at higher frequencies. The reference noise is much greater than the $1 / f$ noise from the LLRF electronics. The $\mathrm{rf}$ station in closed loop with the LLRF controller has an almost flat frequency response and noise power spectrum up to the closed loop bandwidth of the rf feedback $(\approx 300 \mathrm{kHz}$ ). Figure 3 shows the phase noise of the beam 2 cavity sum signal and the VCXO reference (not regulated by the BPL; signal available at the LLRF crate). It is obvious from this figure that the cavity sum noise follows the $400.8 \mathrm{MHz}$ reference up to approximately $300 \mathrm{~Hz}$. At higher frequencies, the noise is dominated by the closed loop rf station noise induced by the LLRF controller. This separation is of course applicable only to this specific technical implementation [8].

Since the BPL acts on the rf reference around the synchrotron frequency and the noise is correlated over all cavities in this frequency range, an improvement of the noise spectrum around $f_{s}$ is anticipated with the BPL closed. Figure 4 shows this phase noise reduction around the synchrotron frequency of about $23 \mathrm{~Hz}$ due to the BPL.

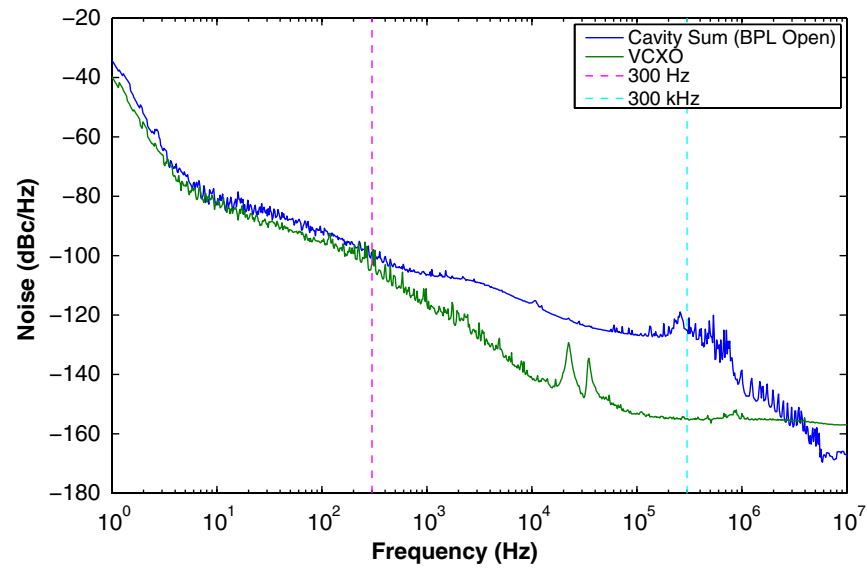

FIG. 3. Phase noise power spectra for beam 2 cavity sum and VCXO (BPL open).

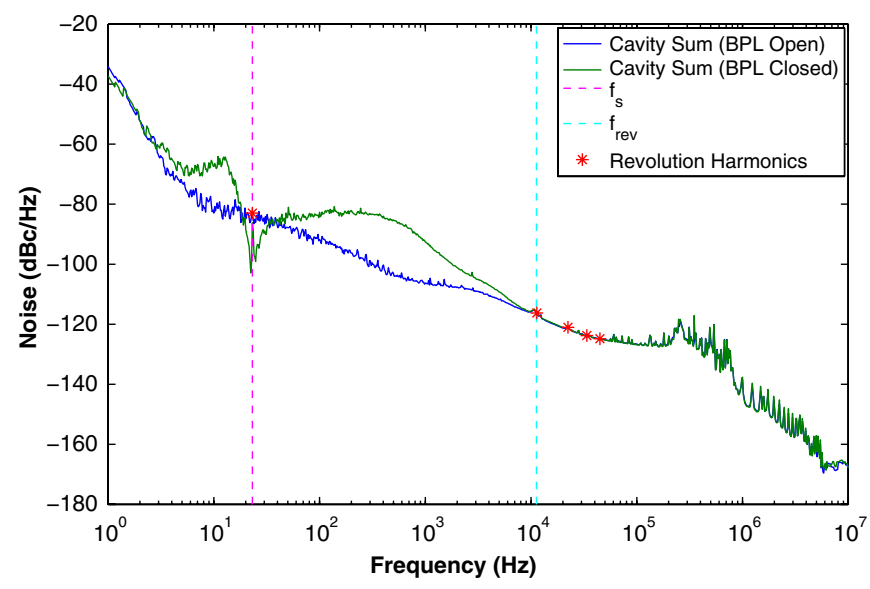

FIG. 4. Beam 2 cavity sum signal with BPL open/closed.

It is interesting to note the increase of the noise levels outside a narrow band around $f_{s}$ and up to the $11.245 \mathrm{kHz}$ bandwidth of the BPL. This increase depends on the BPL gain as will be shown in Sec. V. It is also important to see that the cavity sum signals are identical outside this bandwidth independently of the state of the BPL, as expected since the LLRF noise contribution dominates at these frequencies and its configuration has not changed.

Since the beam is sensitive to the noise power spectral density around $k f_{\text {rev }} \pm f_{s}$, with the BPL open at least $98 \%$ of the beam sampled power $P_{n}$ is attributed to the single contribution at $f_{s}(k=0)$ due to the rf reference. On the other hand, when the BPL is closed, with normal operation gain, the impact of the rf reference noise is insignificant compared to the LLRF levels. The beam sampling frequencies for $k \in[0,4]$ are marked for reference in Fig. 4.

\section{LHC BEAM DIFFUSION MEASUREMENTS}

Dedicated measurements were conducted to better quantify the relationship between the sampled noise power and the bunch length, and also to better understand the effect of the BPL. Measurements were conducted with both protons (May, October 2010) and ions (November 2010). The fundamental difference between those two measurements is that the IBS contributions to beam diffusion are much stronger for ions, since IBS has a $1 / \gamma$ dependence [9].

\section{A. Protons}

During this measurement, the LHC was operating at $3.5 \mathrm{TeV}$, with a noncolliding, single bunch of $9 \times 10^{9}$ intensity per ring. The initial bunch length was approximately $110 \mathrm{ps}$ for both beams. The total rf voltage was set to $8 \mathrm{MV}$. In this study, the BPL gain was varied which had a significant effect on the noise power spectral density around $f_{s}(k=0)$, and consequently the noise power sampled by the beam. The wideband spectral density for rf station 6 of beam 2 ( $r f$ station 6B2) is shown in Fig. 5, as a function of the BPL gain. Figure 6 shows the same 


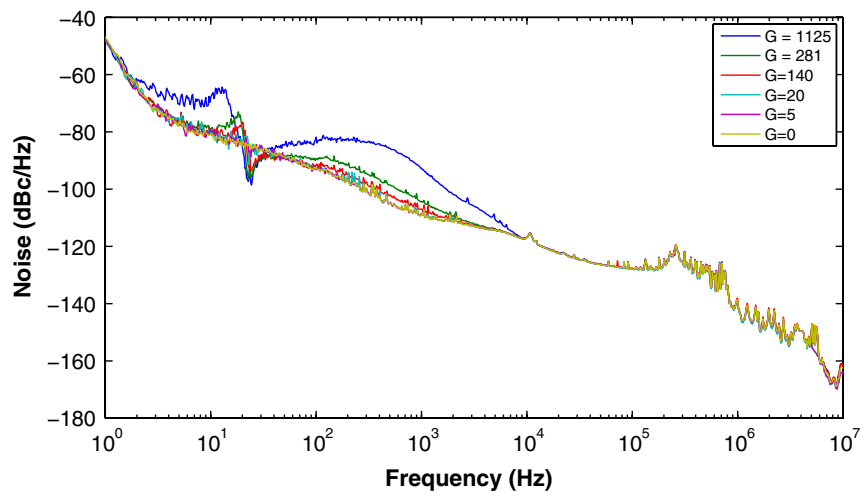

FIG. 5. The rf station 6B2 noise spectral density with BPL gain.

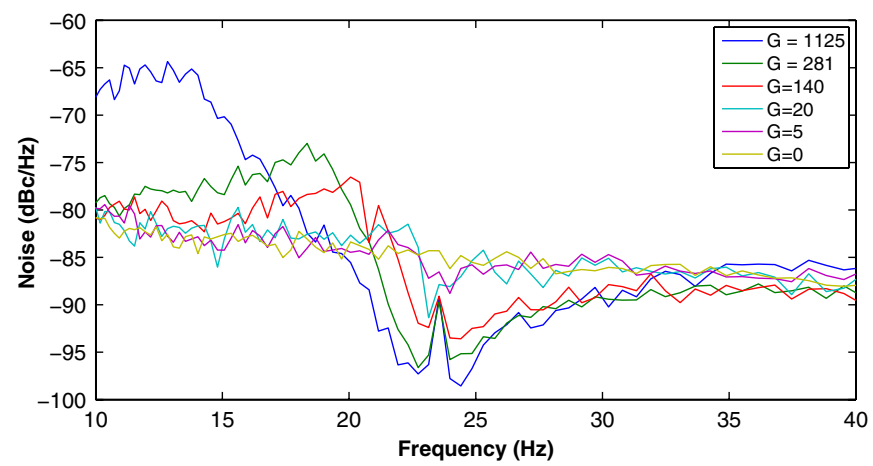

FIG. 6. The rf station 6B2 noise spectral density with BPL gain.

measurement enlarged around $f_{s}$. Increasing the BPL gain clearly decreases the noise at $f_{s}$. The limited instrument resolution is evident in this measurement.

Figures 7 and 8 show the effect of the BPL gain settings on the longitudinal bunch length for beam 1 and beam 2 , respectively. ${ }^{2}$ For high gain settings, the rf noise is low enough that the beam diffusion is dominated by IBS-a 3-4 ps/hr IBS bunch length growth rate is expected for these settings [11,12]. As a result, there is no significant improvement in beam diffusion for a BPL gain of more than approximately $30 \mathrm{~s}^{-1}$.

The growth rate of the longitudinal bunch length can be approximated from these figures. Using Eq. (1), and the measured accelerating voltage noise spectrum, it is then possible to estimate bunch length growth rate for each setting and compare with the measured growth rates. The results are presented in Table I for beam 1 and Table II for beam 2. One can see the clear correlation between the scaled bunch length as estimated by Eq. (1) and the

\footnotetext{
${ }^{2}$ Bunch length data used in this work were provided by the beam quality monitor (BQM) [10]. The BQM employs a fullwidth at half-maximum algorithm to estimate the bunch length from longitudinal pickup data.
}

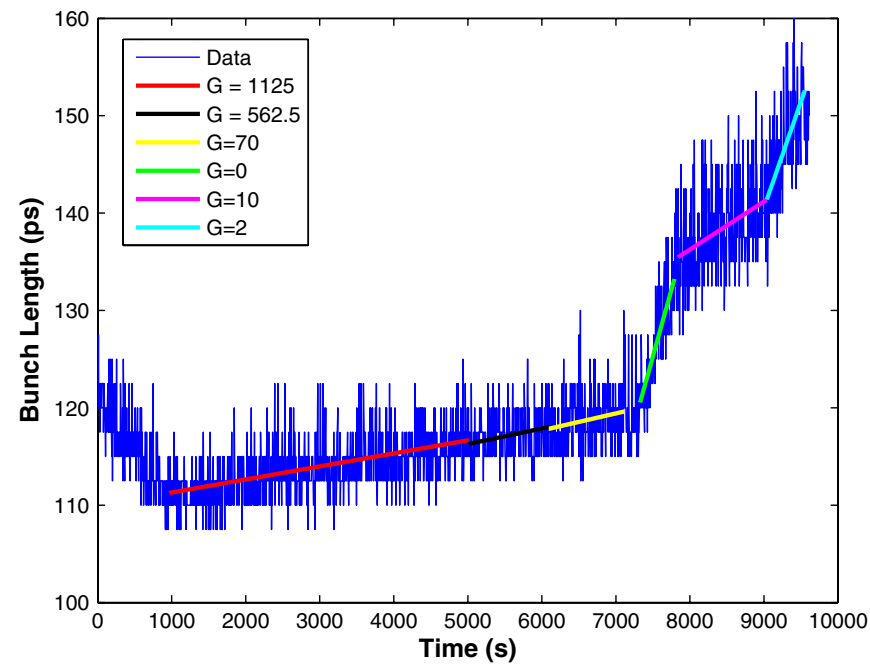

FIG. 7. Beam 1 bunch length with time.

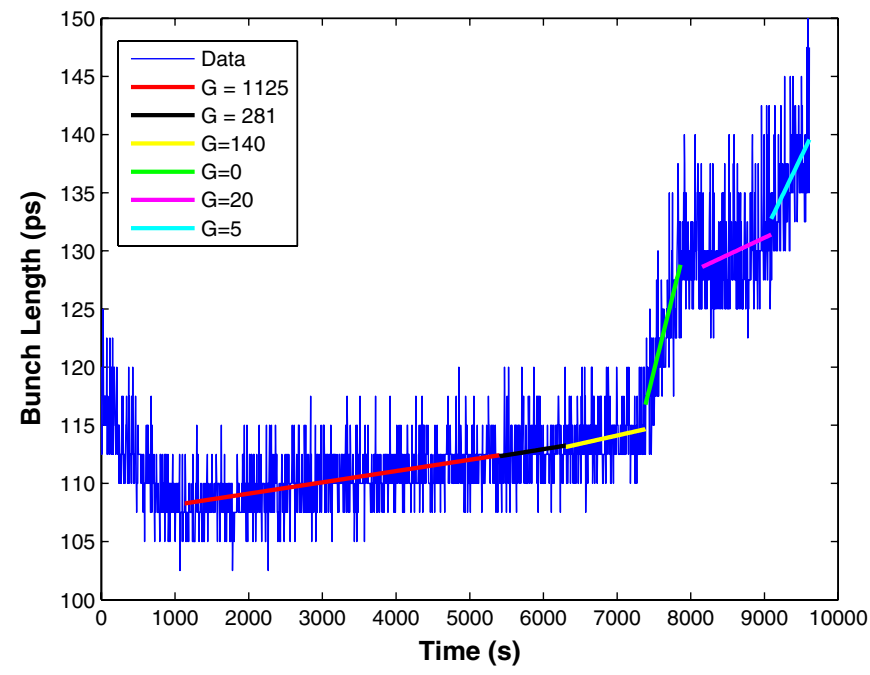

FIG. 8. Beam 2 bunch length with time.

longitudinal emittance growth. As expected, the agreement is better for higher noise levels, since at those points the IBS contributions are insignificant and there is less uncertainty in the bunch length growth estimation. The limited instrument resolution around the very sharp notch in the noise spectrum right at $f_{s}$ as shown in Fig. 6, as well as the growth rate estimate over short time interval restrict the accuracy of this comparison.

The rms rf station phase noise is also included in these tables to show that this is not a valuable metric for beam dynamics performance. The rms rf station phase noise is computed by integrating the phase noise spectrum as shown in Fig. 5. These values show no correlation with the change in bunch length growth and no or little variation with the BPL gain, since the bunch length growth is only sensitive to the magnitude of the rf station phase noise around the synchrotron frequency. 
TABLE I. Bunch growth rate dependence on BPL gain and noise power for B1.

\begin{tabular}{|c|c|c|c|c|}
\hline \multirow[b]{2}{*}{$\begin{array}{l}\text { BPL } \\
\text { gain }\end{array}$} & \multirow{2}{*}{$\begin{array}{c}\text { Estimated } \\
d \sigma_{\tau}^{2} / d t \\
\left(\mathrm{ps}^{2} / \mathrm{hr}\right)\end{array}$} & \multicolumn{2}{|c|}{ Measured } & \multirow{2}{*}{$\begin{array}{c}\text { Integrated } \\
\text { rf noise } \\
(\mathrm{mrad})\end{array}$} \\
\hline & & $\begin{array}{l}d \sigma_{\tau}^{2} / d t \\
\left(\mathrm{ps}^{2} / \mathrm{hr}\right)\end{array}$ & $\begin{array}{c}d \sigma_{\tau} / d t \\
(\mathrm{ps} / \mathrm{hr})\end{array}$ & \\
\hline 0 & 15000 & 25000 & 107 & 1.9 \\
\hline 2 & 10400 & 11300 & 79 & 1.8 \\
\hline 10 & 6600 & 4900 & 18 & 2 \\
\hline 70 & 1000 & 1500 & 6 & 1.9 \\
\hline 562.5 & 700 & 1400 & 6 & 2.2 \\
\hline 1125 & 700 & 1100 & 5 & 3.3 \\
\hline
\end{tabular}

TABLE II. Bunch growth rate dependence on BPL gain and noise power for B2.

\begin{tabular}{|c|c|c|c|c|}
\hline \multirow[b]{2}{*}{$\begin{array}{l}\text { BPL } \\
\text { gain }\end{array}$} & \multirow{2}{*}{$\begin{array}{c}\text { Estimated } \\
d \sigma_{\tau}^{2} / d t \\
\mathrm{ps}^{2} / \mathrm{hr}\end{array}$} & \multicolumn{2}{|c|}{ Measured } & \multirow{2}{*}{$\begin{array}{c}\text { Integrated } \\
\text { rf noise } \\
(\mathrm{mrad})\end{array}$} \\
\hline & & $\begin{array}{c}d \sigma_{\tau}^{2} / d t \\
\mathrm{ps}^{2} / \mathrm{hr}\end{array}$ & $\begin{array}{c}d \sigma_{\tau} / d t \\
(\mathrm{ps} / \mathrm{hr})\end{array}$ & \\
\hline 0 & 19000 & 22500 & 91 & 2.2 \\
\hline 5 & 12800 & 12900 & 47 & 2.1 \\
\hline 20 & 5500 & 2800 & 8 & 2 \\
\hline 140 & 2900 & 1200 & 5 & 2.1 \\
\hline 281 & 1250 & 800 & 4 & 2.2 \\
\hline 1125 & 1000 & 800 & 3 & 3.1 \\
\hline
\end{tabular}

\section{B. Ions}

Through these initial measurements, it was evident that the growth rate of the bunch length is strongly related to the accelerating voltage phase noise power spectral density around $k f_{\text {rev }} \pm f_{s}$, as predicted in [1]. For a more quantitative and accurate study, a first test to inject noise around $f_{s}$ was not conclusive as the BPL significantly modified the injected noise spectrum. Therefore, a technique was developed to inject noise of controllable amplitude in a narrow band around the synchrotron sidebands of a set revolution harmonic ( $k=1$ for these measurements). Measurements were then conducted in November 2010 using ions at $3.5 \mathrm{Z} \mathrm{TeV}$, with four equidistant noncolliding bunches of $7 \times 10^{9}$ intensity per ring. The initial bunch length was approximately $160 \mathrm{ps}$ for both beams. The total rf voltage was set to $12 \mathrm{MV}$. The bunch emittance was blown up transversely to reduce IBS.

Noise was injected in one rf cavity per ring for this measurement, with a bandwidth of $10 \mathrm{~Hz}$ from $f_{s}-$ $10 \mathrm{~Hz}$ to $f_{s}$. The injected noise power level was varied during this measurement. Noise was also injected around the third harmonic of the synchrotron frequency $(m=3)$, to investigate the noise effects on the tails of the bunch distribution. Figure 9 shows the power spectral density of the various levels of injected noise around $f_{\text {rev }} \pm f_{s}$.

Figures 10 and 11 show the resulting bunch length growth for beam 1 and beam 2, respectively, with linear fits for each noise level. The same noise files and levels

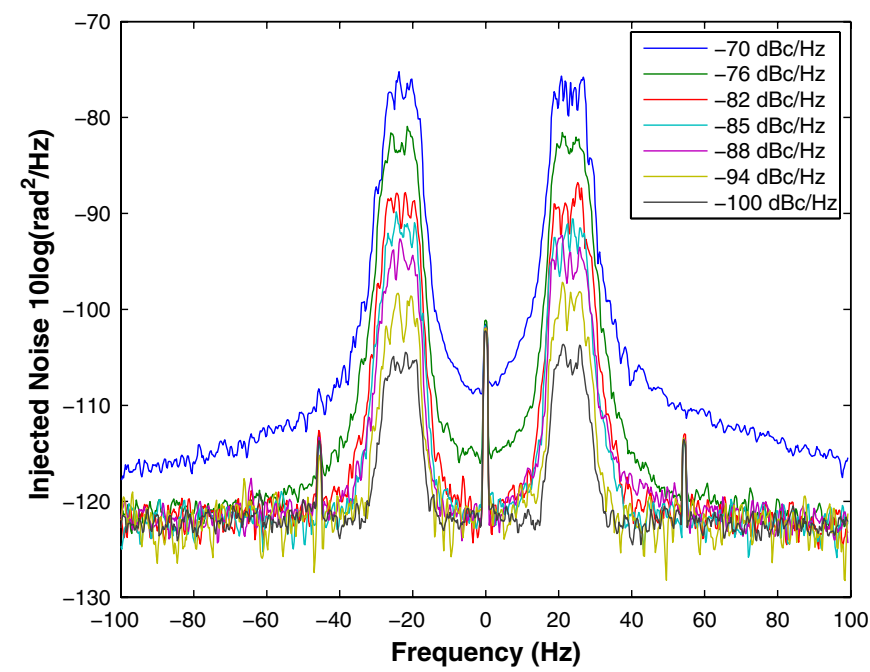

FIG. 9. Levels of injected noise around $f_{\text {rev }} \pm f_{s}$. Horizontal axis shifted by $f_{\text {rf }}+f_{\text {rev }}$.

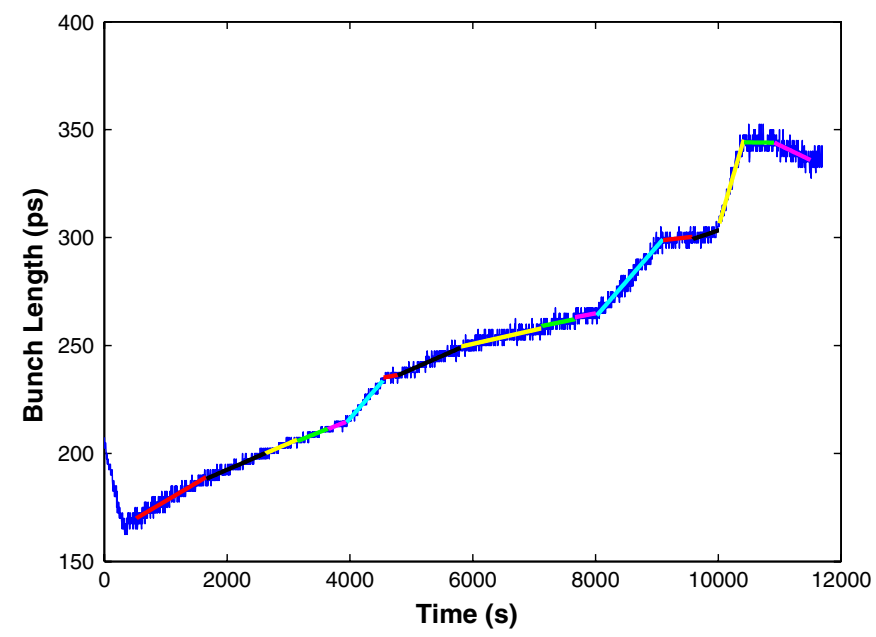

FIG. 10. Beam 1 bunch length growth. Data in blue. Linear segments correspond to different noise injection levels.

were used for both beams. As a result, the bunch length growth was identical for both beams, except for the last part, where the beam losses for beam 2 had become substantial and probably affected the precision of the bunch length estimate. The beam intensity during this measurement is shown in Fig. 12.

The bunch length growth in $\mathrm{ps} / \mathrm{hr}$ can be determined by the slope of the linear fit. Of course, the accuracy of these estimates is limited by the length of each time segment and the granularity of the BQM measurements. Table III shows the results for both beams (the negative numbers in the end are due to the substantial beam loss). In the early stages of this measurement (up to the first $-76 \mathrm{dBc} / \mathrm{Hz}$ level in bold), the bunch is short enough that the growth is dominated by IBS. This background level of about $40 \mathrm{ps} / \mathrm{hr}$ is present until the bunch grows sharply from 210 to $240 \mathrm{ps}$ and is mostly attributed to IBS. As the bunch grows longer, 


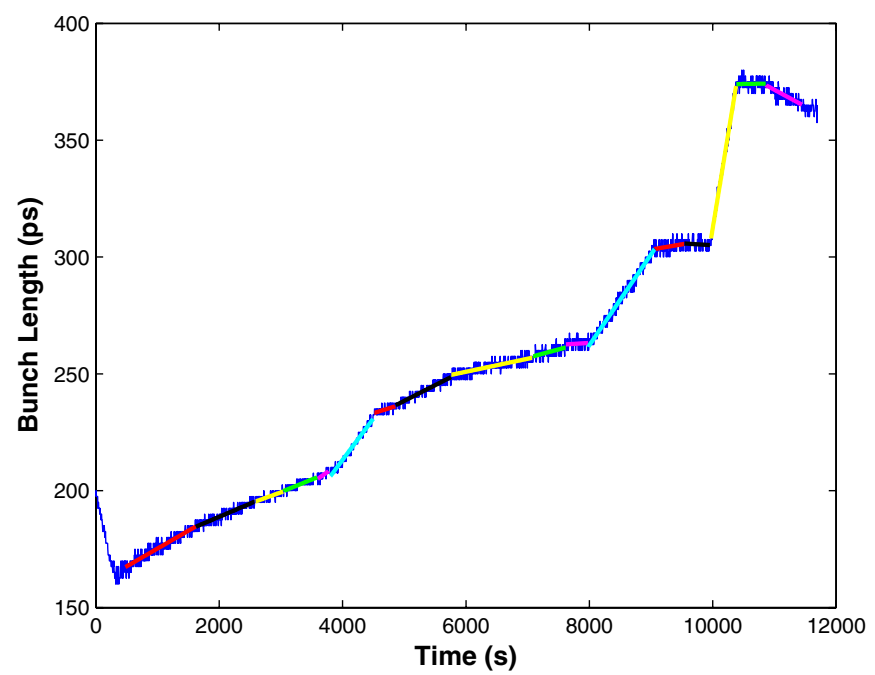

FIG. 11. Beam 2 bunch length growth. Data in blue. Linear segments correspond to different noise injection levels.

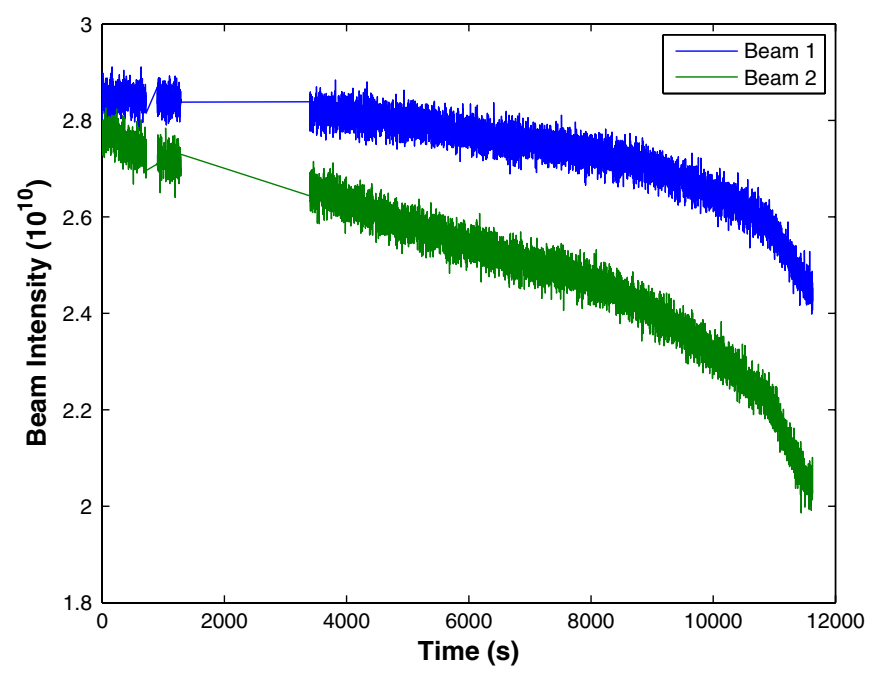

FIG. 12. Beam intensity.

the background growth (from IBS plus the nominal rf noise) drops to about $20 \mathrm{ps} / \mathrm{hr}$. It is also evident that the threshold for the injected noise is between -82 and $-85 \mathrm{dBc} / \mathrm{Hz}$; since in the former case there is no noticeable change from the background level, whereas in the latter there is a measurable increase in the bunch length growth rate.

The estimated growth from Eq. (1) is shown for all the noise levels higher than or comparable to the noise threshold. There is good agreement with the measurements. The reported values are $d \sigma_{\tau} / d t$ rather than $d \sigma_{\tau}^{2} / d t$, so there is an additional dependence to $\sigma_{\tau}$.

The growth rates for beam 2 are slightly larger for this measurement. This discrepancy was traced back to an increased noise level of about $10 \mathrm{~dB}$ for cavity 3 of the beam $2 \mathrm{rf}$ system. Otherwise, the noise spectra are very similar between the two beams.
TABLE III. Bunch length growth as a function of the power spectral density (PSD) of the injected noise-referred to a single sideband (SSB).

\begin{tabular}{lccc}
\hline \hline PSD $\left(10 \log \left(\mathrm{rad}^{2} / \mathrm{Hz}\right)\right)$ & $\begin{array}{c}\mathrm{B} 1 d \sigma_{\tau} / d t \\
(\mathrm{ps} / \mathrm{hr})\end{array}$ & $\begin{array}{c}\mathrm{B} 2 d \sigma_{\tau} / d t \\
(\mathrm{ps} / \mathrm{hr})\end{array}$ & $\begin{array}{c}\text { Estimated } \\
(\mathrm{ps} / \mathrm{hr})\end{array}$ \\
\hline Background & 60 & 72 & \\
-100 & 45 & 40 & \\
-94 & 44 & 34 & \\
-88 & 42 & 39 & \\
-82 & 41 & 68 & 40 \\
$-\mathbf{7 6}$ & $\mathbf{1 1 9}$ & $\mathbf{1 2 8}$ & 150 \\
Background & 19 & 30 & \\
-82 & 45 & 49 & 35 \\
Background & 23 & 20 & \\
-85 & 21 & 26 & 20 \\
Background & 20 & 6 & \\
-76 & 119 & 140 & 125 \\
Background & 12 & 16 & \\
-76 at $3 f_{s}$ & 35 & -6 & \\
-70 & 352 & 580 & $380-450$ \\
Background & -1 & 1 & \\
-70 at $3 f_{s}$ & -49 & -52 & \\
\hline \hline
\end{tabular}

\section{LHC RF NOISE THRESHOLD}

Since the bunch length growth rate $d \sigma_{\tau} / d t$ is approximately proportional to the rf noise power, it is possible to estimate a noise threshold to reach a growth rate of $2.5 \mathrm{ps} / \mathrm{hr}$ (or equivalently a growth rate of $10 \mathrm{ps} / \mathrm{hr}$ of the $4 \sigma_{\tau}$ bunch length). This rate achieves an acceptable lifetime and is comparable to the IBS growth.

At the time of the proton measurements from Sec. VA the noise injection capability was not implemented. As a result, the highest rf noise level for the proton data occurred when the BPL was off, with a SSB noise PSD of approximately $-85 \mathrm{dBc} / \mathrm{Hz}$ at the fundamental band $(k=0)$. In this case, the fundamental band is dominating, so we do not need to include the other contributions at $k f_{\text {rev }} \pm f_{s}$. A bunch length growth rate of about $100 \mathrm{ps} / \mathrm{hr}$ was measured with this configuration. Therefore, to achieve $2.5 \mathrm{ps} / \mathrm{hr}$ the SSB noise power spectral density should be approximately $-101 \mathrm{dBc} / \mathrm{Hz}$. This noise threshold is per cavity and assumes uncorrelated noise sources, based on the analysis in Sec. IV.

During the ion measurements presented in Sec. V B, the injected noise power is larger than the nominal rf station noise, so that the bunch length growth rate $d \sigma_{\tau} / d t$ is approximately proportional to the injected noise power, if the noise is large enough to be the dominant contribution over IBS. For this reason and since the beam loss is not too high to affect the accuracy of the estimate, the SSB PSD level of $-76 \mathrm{dBc} / \mathrm{Hz}$ is used for the noise threshold estimate. At that noise level, the growth rate was estimated to be about $130 \mathrm{ps} / \mathrm{hr}$. Scaling to $2.5 \mathrm{ps} / \mathrm{hr}$, we get a 
threshold of approximately $-93 \mathrm{dBc} / \mathrm{Hz}$ for a single cavity (SSB). The threshold adjusted for all eight rf cavities is then $-102 \mathrm{dBc} / \mathrm{Hz}$. Not surprisingly, the estimates for protons or ions are in close agreement.

The cumulative PSD from the double synchrotron sidebands around each of the 30 revolution harmonics between $f_{\text {rev }}$ and the end of the closed loop cavity bandwidth (approximately $300 \mathrm{kHz}$ ) is approximately $-110 \mathrm{dBc} / \mathrm{Hz}$ according to the LHC measurements. With the BPL on, the noise contribution at the $f_{s}$ is reduced below this level. Therefore, the LHC rf noise is about $9 \mathrm{~dB}$ lower than the noise level for $2.5 \mathrm{ps} / \mathrm{hr}$ growth rate, assuming the current gain and phase settings for the LLRF feedback.

\section{LLRF FEEDBACK NOISE DEPENDENCE ON RF FEEDBACK GAIN SETTINGS}

As the LHC moves to higher energies/intensities, the rf and LLRF configurations will be changed. For example, a new LLRF board will be commissioned (one-turn feedback) to further reduce the cavity effective impedance. These changes and additions may reduce the operational margin estimated above.

Work is in progress to estimate the LHC phase noise levels for future operations using a time-domain simulation of the beam-rf station interaction. It was important to validate the noise levels between the simulation and the physical system first. The LHC time-domain simulations were initially validated through transfer function measurements [2]. A validation of the noise sources and their effect on the accelerating cavity noise spectrum was also necessary. Using the noise levels for the LLRF components (presented in Sec. VIII) in the simulation, it was possible to estimate the LLRF system contribution to the accelerating voltage phase noise spectrum for various LLRF feedback gain settings and then compare with measurements from the physical system. Figure 13 shows the clear

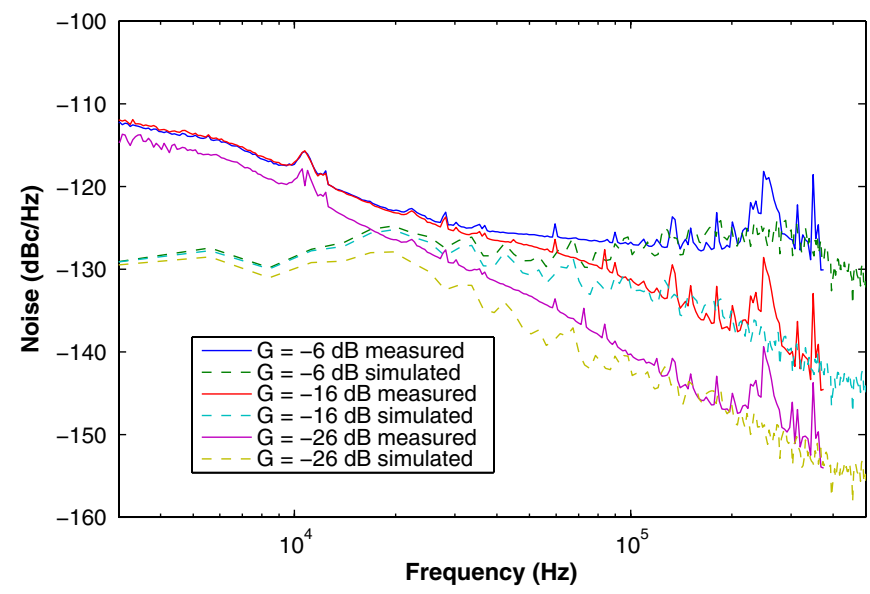

FIG. 13. Accelerating voltage phase power spectral density with controller gain setting from 4B2 (solid lines) and simulation (dashed lines). dependence of the wideband noise spectrum on the LLRF gain settings, as well as the close agreement of the LHC simulations with the measurement for the various gain settings, covering the full scale of operation. Since the $\mathrm{rf}$ reference noise contribution is not modeled in the simulation, there is a discrepancy at frequencies below the revolution frequency where the reference is the dominating noise source. The rf reference contributions are currently added to the simulation. The noise around $240 \mathrm{kHz}$ and other narrow lines at the edge of the bandwidth are introduced by the fiber optic transmitter and receiver used in the rf reference distribution and have been corrected for 2011 operations.

It is obvious from this figure that the simulation provides a good representation of the noise power spectrum of the $\mathrm{rf}$ station for frequencies higher than a few kHz. As such, it can be very useful in predicting the system behavior for various rf configurations, estimating the coupled-bunch instabilities, and determining the contribution of the phase noise to beam diffusion around $k f_{\text {rev }} \pm f_{s}$ for $k>0$.

\section{LLRF FEEDBACK NOISE}

As the LHC approaches nominal energies and currents, it is conceivable that the LLRF contributions to rf noise will exceed the noise threshold set in this work. Therefore, a better understanding of the LLRF feedback noise contributions is essential.

The LLRF feedback modules are simplified in the block diagram shown in Fig. 14. There are two main modules, the analog and digital module. Each module includes a demodulator to transform the rf cavity signal to baseband and an attenuator to adjust the controller gain. In the digital module, the processing includes an analog to digital converter (ADC) and a low-pass filter implemented in a fieldprogramable gate array. Because of the limited rf input range of the demodulators, the high level rf cavity signal is attenuated significantly before the demodulation. To recover the loop gain, a large gain stage follows the demodulation.

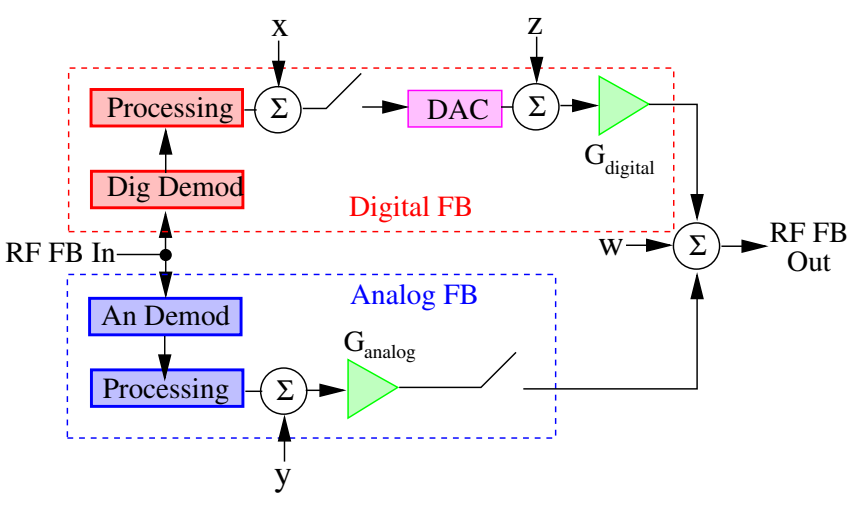

FIG. 14. The rf feedback block diagram. 
TABLE IV. Component wise contribution in $\mathrm{mV}$ rms.

\begin{tabular}{lccc}
\hline \hline Digital $(x)$ & Analog $(y)$ & DAC $(z)$ & Backend $(w)$ \\
\hline 2.2 & 3.6 & 0.25 & 0.28 \\
\hline \hline
\end{tabular}

There are multiple electronic components with very diverse noise specifications. For this analysis and due to the topology of the switches, all the LLRF noise sources are referred to four groups: the analog feedback path with rms noise $y$, the digital feedback path up to the switch with rms noise $x$, the digital to analog converter (DAC) with rms noise $z$, and the backend processing (summing junction and amplifiers) with rms noise $w$. Based on this model, the total noise power $N^{2}$ can be modeled as

$$
N^{2}=G_{\text {analog }}^{2} y^{2}+G_{\text {digital }}^{2}\left(x^{2}+z^{2}\right)+w^{2} .
$$

By terminating the input of the rf feedback, switching the analog and/or digital path on and off, and adjusting the gains $G_{\text {analog }}$ and $G_{\text {digital }}$, it is possible to determine the contributions from each one of these four components. Table IV presents the estimates from these measurements. It is obvious that the digital and analog modules dominate the noise contributions. The data shown in Fig. 15 qualitatively confirm the measurements from Table IV. It shows the noise spectrum at the rf feedback output with different switch positions when the input is terminated. The noise level is significantly lower when both the analog and digital path are turned off. This figure also shows the $1 / f$ noise from the LLRF electronics.

Similar values were estimated by cascading the noise contributions of the electronics from the detailed layouts. This study also identified the most significant electronics components for the noise characteristics of each of the two dominant modules. The dominant components on the digital path are the differential amplifier driving the ADC, and the digitizing noise of the ADC. For the analog path of the rf feedback the noise level is dominated by the noise contribution of the large amplification after the analog demodulator. ${ }^{3}$

It is expected that as the controller gain is reduced, the noise levels would be initially reduced almost linearly with gain. As the gain gets lower though, the backend components would start dominating. This effect is visible in Fig. 16 where the controller gain is set to $0,10,20$, and $31 \mathrm{~dB}$ and the rf feedback input is terminated.

These studies provide insight on the performance of the existing LHC LLRF system and the tools to study the effect of future rf and LLRF implementations on beam diffusion. Furthermore, this insight on the interplay

\footnotetext{
${ }^{3}$ Figure 1 shows a single demodulator for simplicity, but in the actual implementation there are two demodulators (analog and digital channels), as shown in Fig. 14.
}

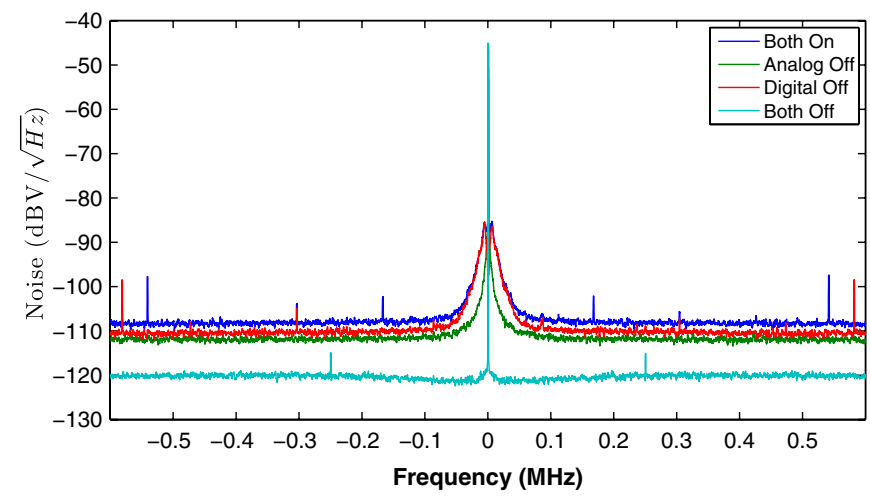

FIG. 15. Noise PSD at the rf feedback output with different switch positions. The rf input is terminated.

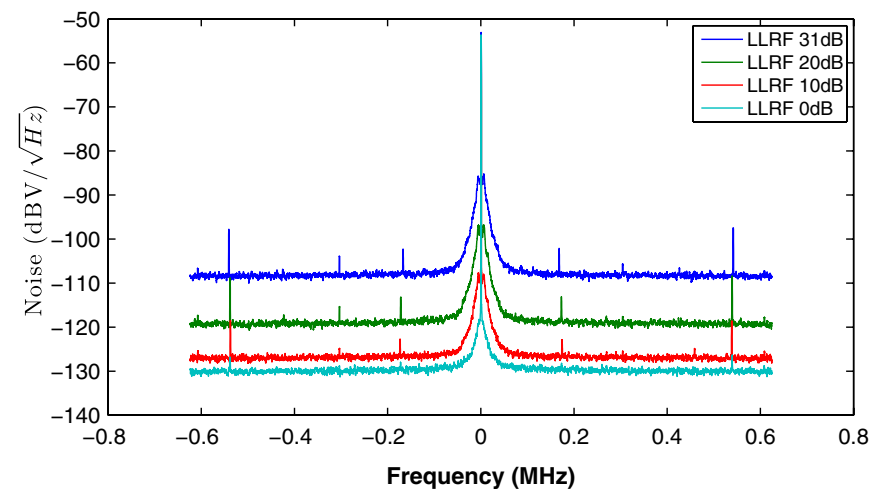

FIG. 16. Noise PSD at the rf feedback output as a function of controller gain. The rf input is terminated.

between topology and component specifications will be very useful for noise budgeting in future LLRF systems.

\section{CONCLUSIONS AND FUTURE DIRECTIONS}

Dedicated measurements were conducted in the LHC to gain insight in the effect of rf noise on the longitudinal beam diffusion. It was evident that the growth rate of the bunch length is strongly related to the accelerating voltage phase noise power spectral density around $k f_{\text {rev }} \pm f_{s}$, as predicted in [1]. When the BPL is off, the noise power is dominated by a single contribution at $f_{s}$, which depends strongly on the $400.8 \mathrm{MHz}$ reference noise. With the BPL on, the noise power level is set by the LLRF contributions. The elements that influence the noise spectrum of the $\mathrm{rf}$ feedback system were identified. It was also shown that the time-domain simulation presented in [2] provides a close representation of the rf system behavior.

The noise threshold for $2.5 \mathrm{ps} / \mathrm{hr}$ growth was estimated to be $-101 \mathrm{dBc} / \mathrm{Hz}$ (SSB flat noise spectral density from $f_{s}$ to the edge of the closed loop bandwidth). A $9 \mathrm{~dB}$ margin is achieved with the current rf configuration and the BPL on. Work is in progress to use these measurements, analysis, and noise thresholds to estimate the bunch 
length growth rate for future rf and LLRF settings and configurations.

The work and analysis presented in this work uses the bunch length as the primary metric of beam diffusion. As the bunch distribution deviates from a Gaussian, this description becomes less accurate. Work is in progress to relate the measured cavity noise spectrum with a diffusion coefficient and subsequently with the evolution of the beam distribution.

\section{ACKNOWLEDGMENTS}

The authors would like to thank the CERN BE-RF group for their help, support, and interest in all phases of this project. We are grateful to Elena Shaposhnikova for her insight and guidance during the beam diffusion measurements. The authors would also like to thank Joachim Tuckmantel at CERN for all his inspiring work on beam diffusion effects. Conversations with Ron Ruth at SLAC were very helpful for the development of the single bunch longitudinal beam emittance growth formalism. The USLARP program and SLAC AARD group have greatly supported this work. This work was supported by the DOE through the U.S. LHC Accelerator Research Program (LARP) and under Contract No. DE-AC0276 SF00515.
[1] T. Mastorides et al., Phys. Rev. ST Accel. Beams 13, 102801 (2010).

[2] T. Mastorides et al., in Proceedings of the 11th European Particle Accelerator Conference, Genoa, 2008 (EPS-AG, Genoa, Italy, 2008).

[3] T. Mastorides et al., in Proceedings of the IPAC'10 Conference, Kyoto, Japan (ICR, Kyoto, 2010).

[4] J. Tuckmantel, LHC Project Report No. 819, 2005.

[5] Th. Leiber et al., Phys. Rev. Lett. 59, 1381 (1987).

[6] D. Boussard, CERN Report No. CERN 84-15, 1984, p. 283.

[7] J. Steimel et al., in Proceedings of the 20th Particle Accelerator Conference, Portland, OR, 2003 (IEEE, New York, 2003).

[8] P. Baudrenghien et al., in Proceedings of the 10th European Particle Accelerator Conference, Edinburgh, Scotland, 2006 (EPS-AG, Edinburgh, Scotland, 2006).

[9] A. Piwinski, in Proceedings of the 9th International Conference on High Energy Accelerators, Stanford, 1974 (Hamburg Desy Internal Report No. H1-73-30, 1974).

[10] G. Papotti (private communication).

[11] R. Assmann et al., in Proceedings of the 2010 Evian Workshop on LHC Commissioning, Evian, France (CERN, Geneva, 2010).

[12] LHC Design Report No. CERN-2004-003, 2004, Vol. 1, Chap. 2. 